\title{
De Novo Aneurysm Formation Following Gamma Knife Surgery for Arteriovenous Malformation: A Case Report
}

\author{
Takuya Akai ${ }^{1}$ Keiichiro Torigoe ${ }^{1}$ Manna Fukushima ${ }^{2}$ Hideaki lizuka $^{1}$ Yasuhiko Hayashi ${ }^{3}$ \\ ${ }^{1}$ Department of Neurosurgery, Kanazawa Medical University, \\ Kanazawa, Japan \\ 2 Pathology and Laboratory Medicine, Kanazawa Medical University, \\ Address for correspondence Takuya Akai, MD, PhD, 1-1 Daigaku, \\ Uchinada, Kahoku-gun, Ishikawa, 920-0293, Japan \\ Kanazawa, Japan \\ ${ }^{3}$ Department of Neurosurgery, Kanazawa University, Kanazawa, Japan \\ (e-mail: akai@kanazawa-med.ac.jp). \\ J Neurol Surg Rep 2015;76:e105-e108.
}

\begin{abstract}
Background Stereotactic radiosurgery plays a critical role in the treatment of central nervous system neoplasm and cerebrovascular malformations. This procedure is purportedly less invasive, but problems occurring later including tumor formation, necrosis, and vasculopathy-related diseases have been reported.

Clinical Presentation We report on a 65-year-old man who had experienced a de novo aneurysm in an irradiated field and an acute onset of right hemiparesis and aphasia. He had undergone gamma knife radiosurgery to treat an arteriovenous malformation 15 and 12 years prior, with 18 and 22 Gy marginal doses. At current admission, radiologic studies showed a de novo aneurysm in the irradiated field without recurrence of malformation. The aneurysm was resected. Histologic findings showed a disruption of

Keywords

- aneurysm

- radiosurgery

- de novo the internal elastic lamina accompanied by fibrous degeneration.

Conclusion Stereotactic radiosurgery is a promising treatment tool, but long-term risks have not been fully researched. The treatment procedure for benign lesions should be chosen prudently.
\end{abstract}

\section{Introduction}

Stereotactic radiosurgery is an established treatment option for arteriovenous malformation (AVM), benign and malignant brain tumors, and neuralgia because of its efficacy and minimal invasiveness. Conventional radiation-induced late side effects such as tumor formation and vasculopathy-related lesions are well described. ${ }^{1-3}$ However, the occurrence of late complications induced by stereotactic radiosurgery has not been well documented, and research is ongoing. Stereotactic radiosurgery is frequently used for patients with nonmalignant diseases who are expected to have long lifespans, so a radiation-induced problem occurring after radiosurgery is problematic and increases the amount of care such patients would need. We present a patient who experienced epilepsy

received

September 22, 2014 accepted after revision January 13, 2015

published online

May 22, 2015 DOI http://dx.doi.org/
10.1055/s-0035-1549223.
ISSN 2193-6366. due to progressive enlargement of a pseudoaneurysm that seemed to have been induced by gamma knife surgery for an AVM.

\section{Case Report}

A 65-year-old man had an acute onset of right hemiparesis, aphasia, and consciousness disturbance at home. After transfer to the hospital, these symptoms improved within 24 hours. Emergent computed tomography (CT) images showed a $1.5-\mathrm{cm}$ diameter ring-like high-density area in the left Sylvian fissure with a surrounding low-density area that indicated the probable presence of a brain edema (-Fig. 1A). Magnetic resonance (MR) images demonstrated

\footnotetext{
(C) 2015 Georg Thieme Verlag KC Stuttgart · New York
}

License terms

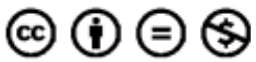



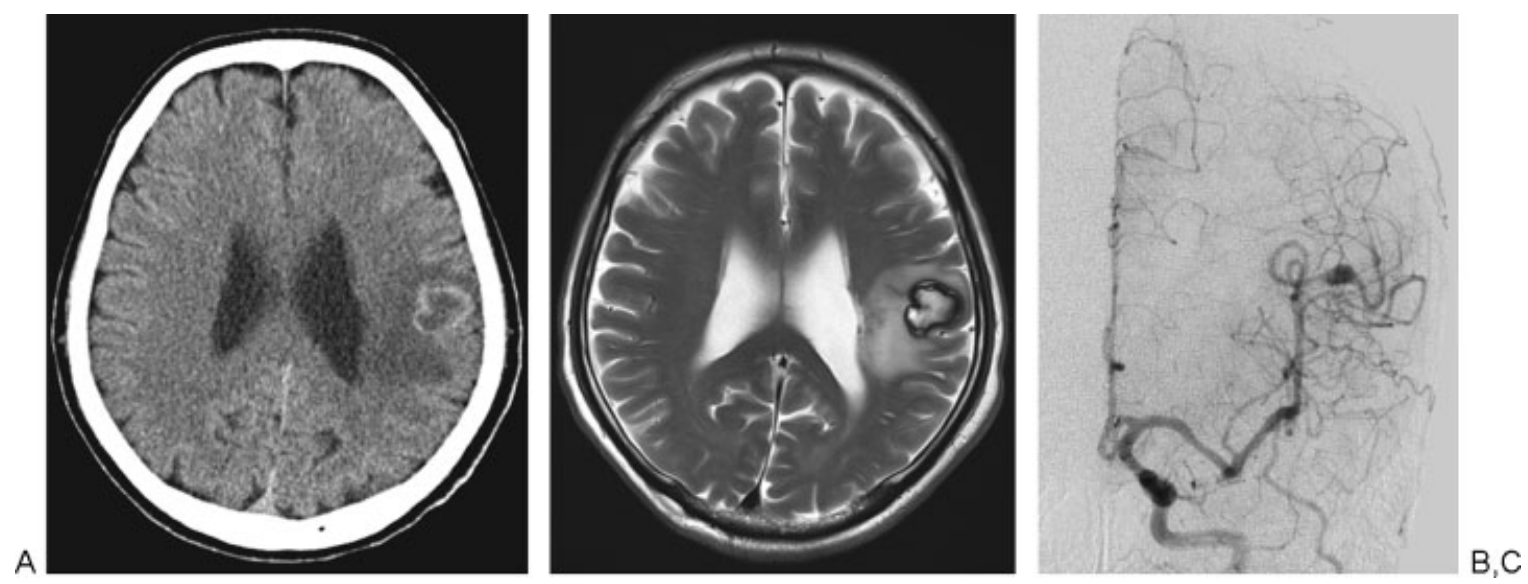

Fig. 1 (A) Computed tomography at admission showed ring-like high density lesion in the left temporal portion. (B) T2-weighted magnetic resonance image showed a high hyperintensity mass with hypointensity rim associated with brain edema. (C) Anteroposterior image of the left carotid angiogram showed a fusiform aneurysm on the left middle cerebral artery.

that the lesion was hyperintense on T1- and T2-weighted images with a hypointense rim with surrounding edema (-Fig. 1B). Angiograms showed an aneurysm on the middle cerebral artery consistent with the lesion demonstrated on CT and MR images (-Fig. 1C).

This patient had a history of gamma knife treatment for an AVM ( - Fig. 2A) on the left middle cerebral artery 15 years before with a marginal dose of $18 \mathrm{~Gy}$. Three years later he received a second gamma knife treatment with a marginal dose of $22 \mathrm{~Gy}$ against the residual nidus. Six years after the initial treatment, the obliteration of the AVM was confirmed by angiograms (-Fig. 2B), and the patient had no further follow-up.

The aneurysm was located on the nonbranching portion of the artery in the irradiated field. From these findings, we diagnosed that this patient had a seizure due to the mass effect of the radiation-induced de novo aneurysm. We resected the aneurysm under somatosensory and motor evoked potentials. The arachnoid membrane surrounding the aneurysm showed thickening and had changed to a white color. The aneurysm was located in the Sylvian fissure and was a fusiform shape ( - Fig. 3A). He showed no new neurologic deficit after the operation.

The resected aneurysm showed that the wall had intimal thickening with inflammatory cell infiltration and fibrous degeneration. The elastic lamina was degenerated and disrupted ( - Fig. 3B-D). The seizure was controlled with antiepilepsy medication, and the patient was discharged without neurologic deficits.

\section{Discussion}

Stereotactic radiosurgery is purported to be a less invasive procedure for vascular malformations and central nervous
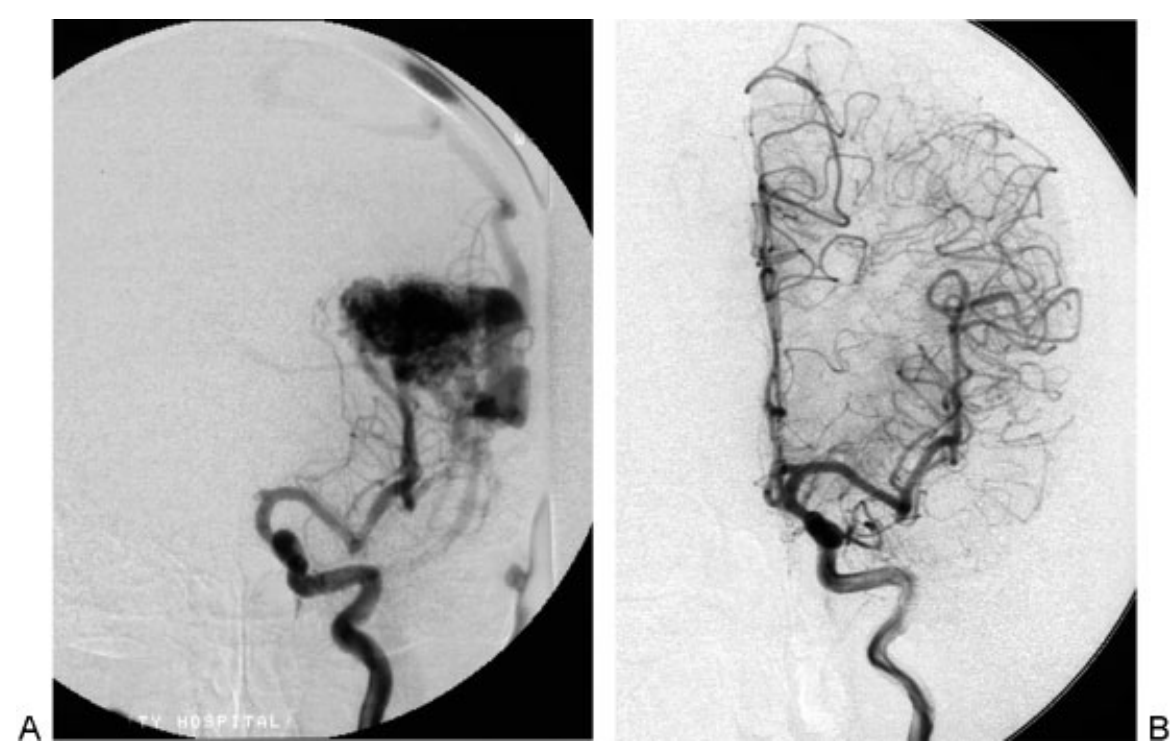

Fig. 2 (A) The anteroposterior image of the left internal carotid angiogram prior to the first radiosurgery showed an arteriovenous malformation fed by the middle cerebral artery and drained into the superior sagittal sinus. (B) The left internal carotid angiogram 3 years after the second radiosurgery showed complete eradication of the shunt flow. 

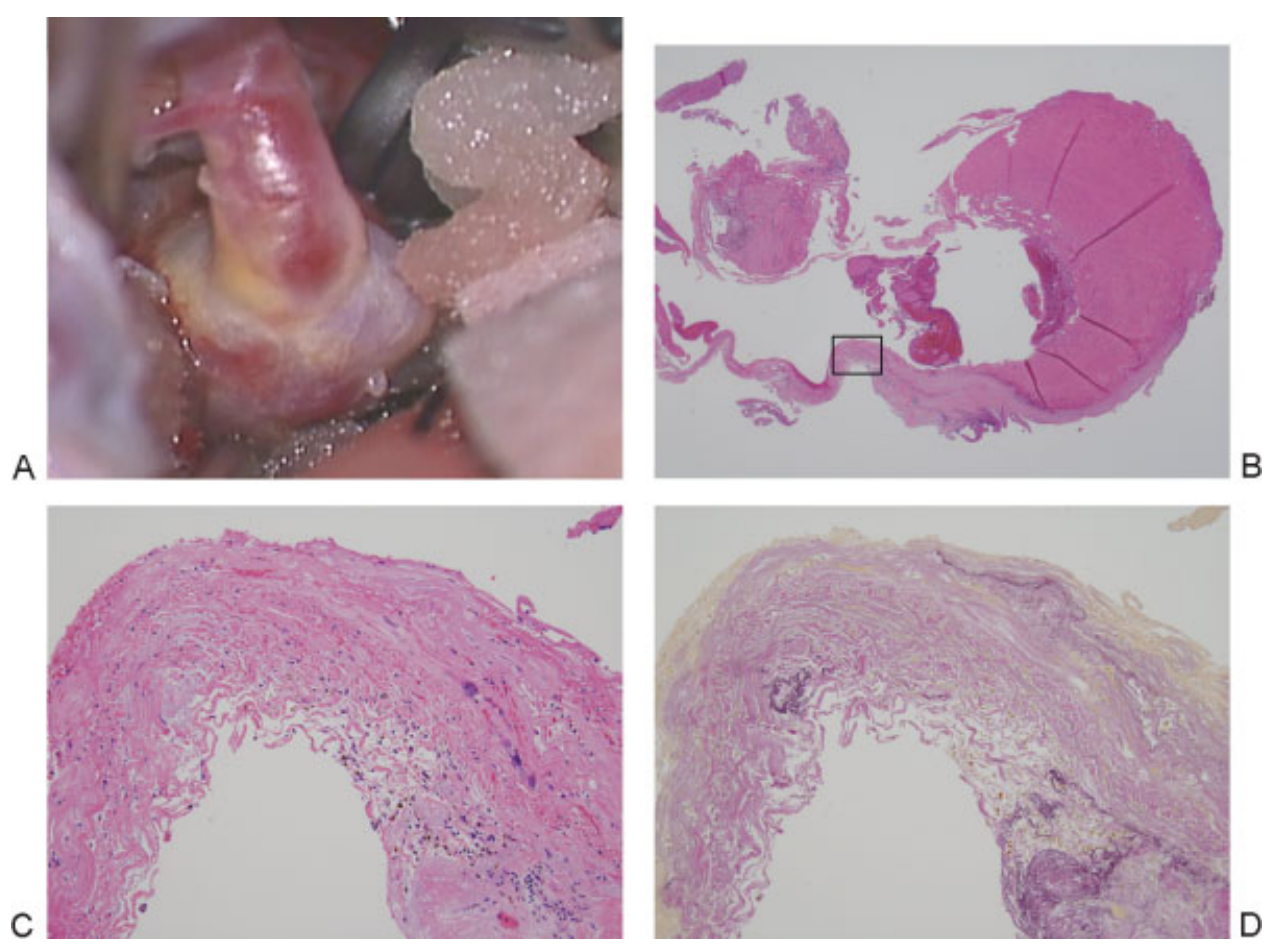

Fig. 3 (A) The aneurysm was located on the nonbranching portion of the artery in the Sylvian fissure. (B, C) The wall of the resected aneurysm showed intimal thickening and fibrous degeneration with inflammatory cell infiltration and calcification (hematoxylin and eosin stain). (D) The internal elastic lamina was degenerated and disrupted (Elastica van Gieson stain). (C, D) Increased magnification of the outlined area in (B). Original magnification $\times 12.5(B), \times 40(C, D)$.

system tumors. For AVMs, the vessels are the main target of radiation; for tumors, vessels should be out of the radiation field. Still, it can induce tumors ${ }^{4-6}$ and vasculopathyrelated lesions such as cysts, ${ }^{7,8}$ vessel occlusions, ${ }^{9}$ and aneurysms. ${ }^{10-15}$

Sciubba et al reported on 26 patients with conventional radiation-induced aneurysms. ${ }^{16}$ For stereotactic radiosurgery, only seven patients including the present patient have

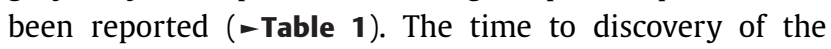
aneurysm after radiation was from 7 months to 29 years (average: 10.7 years) in the conventional group and 9 months to 15 years (average: 7.8 years) in the radiosurgery group. In the radiosurgery group, the original diseases were four cases of vestibular schwannomas, one case of cerebellopontine meningioma, and two cases of AVM. And all of these aneurysms were located in the irradiation field and nonbranching sites. The radiation doses were 12 or $25 \mathrm{~Gy}$ for the schwannomas, $16 \mathrm{~Gy}$ for the meningioma, and 20 and 40 Gy for AVMs.

Radiosurgery damages the endothelial cells and causes proliferation of smooth muscle cells, leading to intimal thickening. It also causes adventitial fibrosis. As the degeneration progresses, this induces vessel wall hyalinization, calcification, and necrosis associated with fragmentation

Table 1 Reported cases of de novo intracranial aneurysms following stereotactic radiosurgery

\begin{tabular}{|c|c|c|c|c|c|c|}
\hline Study & Age/Sex & Original lesion & Dose & SAH & Location & Duration $^{a}$ \\
\hline Huang et al ${ }^{10}$ & $19 / F$ & AVM & 20 Gy & - & Distal ACA & $9 \mathrm{mo}$ \\
\hline Takao et al ${ }^{11}$ & $63 / F$ & Acoustic neuroma & 12 Gy & + & Distal AICA & $6 y$ \\
\hline Akamatsu et al $^{12}$ & $75 / F$ & Acoustic neuroma & $12 \mathrm{~Gy}$ & + & Distal AICA & $8 \mathrm{y}$ \\
\hline Park et al $^{13}$ & $69 / F$ & Acoustic neuroma & 12 Gy & + & Distal AICA & $5 y$ \\
\hline Kellner et al ${ }^{14}$ & $58 / \mathrm{F}$ & Cerebellopontine meningioma & 16 Gy & - & Distal SCA & $10 \mathrm{y}$ \\
\hline Sunderland et al ${ }^{15}$ & $50 / \mathrm{F}$ & Vestibular schwannoma & $13+12$ Gy & + & Distal AICA & $10 \mathrm{y}$ \\
\hline Present case & $65 / \mathrm{M}$ & AVM & $18+22$ Gy & - & Distal MCA & $15 \mathrm{y}$ \\
\hline
\end{tabular}

Abbreviations: ACA, anterior cerebral artery; AICA, anterior inferior cerebellar artery; AVM, arteriovenous malformation; F, female; M, male; MCA, middle cerebellar artery; SAH, subarachnoid hemorrhage; SCA, superior cerebellar artery.

a Time to aneurysm discovery after radiation.

For the de novo intracranial aneurysm following stereotactic radiosurgery, only 7 patients including the present patient have been reported. The original diseases were four cases of acoustic neuroma, two cases of AVM, and one case of meningioma, and the radiation dose was 12 to 40 Gy. In four patients, the aneurysms were found due to the rupture. The time to discovery of the aneurysm after radiosurgery was 9 months to 15 years. 
of the elastic lamina, resulting in occlusion of blood vessels. $^{17-21}$

In the experimental model, endothelial cell proliferation started at 3 hours after irradiation, and the endothelial hyperplasia and vessel wall thickening continued throughout the observation period (90 days after irradiation). ${ }^{20}$ Radiation-induced histologic changes of vessels are supposed to be dose related, and high-dose radiation can induce radionecrosis, ${ }^{18,22}$ but the radiation dose threshold below which no changes are induced is not yet known. ${ }^{18}$

The reported radiation-induced aneurysm was located on the nonbranching portion, ${ }^{10-15}$ and the resected aneurysm wall showed the disruption of the elastic lamina. ${ }^{12}$ The histologic findings in our case were consistent with these findings. We do not know the critical radiation dose that induces an aneurysm formation, but the degeneration of the elastic lamina may play an important role.

\section{Conclusion}

Stereotactic radiosurgery is an established treatment tool for central nervous system lesions and has been frequently used to treat AVMs and benign tumors. Patients who are normally expected to have a long lifespan can run risks of aneurysm formation, vessel occlusion, tumor synthesis, and other unknown late perils. Further research of these late-occurring health problems is needed to identify unknown long-term risks and clarify optimal radiation doses and follow-up periods. The current report also suggests that the treatment procedure be selected prudently, especially among younger patients.

\section{References}

1 Valk PE, Dillon WP. Radiation injury of the brain. AJNR Am J Neuroradiol 1991;12(1):45-62

2 Rabin BM, Meyer JR, Berlin JW, Marymount MH, Palka PS, Russell EJ. Radiation-induced changes in the central nervous system and head and neck. Radiographics 1996;16(5):1055-1072

3 O'Connor MM, Mayberg MR. Effects of radiation on cerebral vasculature: a review. Neurosurgery 2000;46(1):138-149; discussion 150-151

4 Shamisa A, Bance M, Nag S, et al. Glioblastoma multiforme occurring in a patient treated with gamma knife surgery. Case report and review of the literature. J Neurosurg 2001;94(5): 816-821

5 Kaido T, Hoshida T, Uranishi R, et al. Radiosurgery-induced brain tumor. Case report. J Neurosurg 2001;95(4):710-713

6 Berman EL, Eade TN, Brown D, et al. Radiation-induced tumor after stereotactic radiosurgery for an arteriovenous malformation: case report. Neurosurgery 2007;61(5):E1099; discussion E1099
7 Shuto T, Matsunaga S, Suenaga J. Surgical treatment for late complications following gamma knife surgery for arteriovenous malformations. Stereotact Funct Neurosurg 2011;89(2):96-102

8 Shuto T, Ohtake M, Matsunaga S. Proposed mechanism for cyst formation and enlargement following Gamma Knife Surgery for arteriovenous malformations. J Neurosurg 2012;117(Suppl):135-143

9 Yamamoto M, Ide M, Jimbo M, Ono Y. Middle cerebral artery stenosis caused by relatively low-dose irradiation with stereotactic radiosurgery for cerebral arteriovenous malformations: case report. Neurosurgery 1997;41(2):474-477; discussion 477-478

10 Huang PP, Kamiryo T, Nelson PK. De novo aneurysm formation after stereotactic radiosurgery of a residual arteriovenous malformation: case report. AJNR Am J Neuroradiol 2001;22(7): 1346-1348

11 Takao T, Fukuda M, Kawaguchi T, et al. Ruptured intracranial aneurysm following gamma knife surgery for acoustic neuroma. Acta Neurochir (Wien) 2006;148(12):1317-1318; discussion 1318

12 Akamatsu Y, Sugawara T, Mikawa S, et al. Ruptured pseudoaneurysm following Gamma Knife surgery for a vestibular schwannoma. J Neurosurg 2009;110(3):543-546

13 Park KY, Ahn JY, Lee JW, Chang JH, Huh SK. De novo intracranial aneurysm formation after Gamma Knife radiosurgery for vestibular schwannoma. J Neurosurg 2009;110(3):540-542

14 Kellner CP, McDowell MM, Connolly ES, et al. Late onset aneurysm development following radiosurgical obliteration of a cerebellopontine angle meningioma. BMJ Case Rep 2014; May 14 (Epub ahead of print)

15 Sunderland G, Hassan F, Bhatnagar P, et al. Development of anterior inferior cerebellar artery pseudoaneurysm after gamma knife surgery for vestibular schwannoma. A case report and review of the literature. Br J Neurosurg 2014;28(4):536-538

16 Sciubba DM, Gallia GL, Recinos P, Garonzik IM, Clatterbuck RE. Intracranial aneurysm following radiation therapy during childhood for a brain tumor. Case report and review of the literature. J Neurosurg 2006;105(2, Suppl):134-139

17 Yamamoto M, Jimbo M, Ide M, et al. Gamma knife radiosurgery for cerebral arteriovenous malformations: an autopsy report focusing on irradiation-induced changes observed in nidus-unrelated arteries. Surg Neurol 1995;44(5):421-427

18 Chang SD, Shuster DL, Steinberg GK, Levy RP, Frankel K. Stereotactic radiosurgery of arteriovenous malformations: pathologic changes in resected tissue. Clin Neuropathol 1997;16(2): 111-116

19 Schneider BF, Eberhard DA, Steiner LE. Histopathology of arteriovenous malformations after gamma knife radiosurgery. J Neurosurg 1997;87(3):352-357

20 Yang T, Wu SL, Liang JC, Rao ZR, Ju G. Time-dependent astroglial changes after gamma knife radiosurgery in the rat forebrain. Neurosurgery 2000;47(2):407-415; discussion 415-416

21 Jahan R, Solberg TD, Lee D, et al. An arteriovenous malformation model for stereotactic radiosurgery research. Neurosurgery 2007; 61(1):152-159; discussion 159

22 Lunsford LD, Altschuler EM, Flickinger JC, Wu A, Martinez AJ. In vivo biological effects of stereotactic radiosurgery: a primate model. Neurosurgery 1990;27(3):373-382 